\title{
Prehospital care delivery and triage of stroke with emergent large vessel occlusion (ELVO): report of the Standards and Guidelines Committee of the Society of Neurointerventional Surgery
}

\author{
G Lee Pride, ${ }^{1}$ Justin F Fraser, ${ }^{2}$ Rishi Gupta, ${ }^{3}$ Mark J Alberts, ${ }^{4}$ J Neal Rutledge, ${ }^{5}$ \\ Ray Fowler, ${ }^{6}$ Sameer A Ansari, ${ }^{7}$ Todd Abruzzo, ${ }^{8}$ Barb Albani, ${ }^{9}$ Adam Arthur, ${ }^{10}$ \\ Blaise Baxter, ${ }^{11}$ Ketan R Bulsara, ${ }^{12}$ Michael Chen, ${ }^{13}$ Josser E Delgado Almandoz, ${ }^{14}$ \\ Chirag D Gandhi, ${ }^{15}$ Don Heck, ${ }^{16}$ Steven W Hetts, ${ }^{17}$ Joshua A Hirsch, ${ }^{18}$ \\ M Shazam Hussain, ${ }^{19}$ Richard Klucznik, ${ }^{20}$ Seon-Kyu Lee, ${ }^{21}$ William J Mack, ${ }_{1}^{22}$ \\ Thabele Leslie-Mazwi, ${ }^{23}$ Ryan A McTaggart, ${ }^{24}$ Philip M Meyers, ${ }^{25} \mathrm{~J}$ Mocco, ${ }^{26}$ \\ Charles Prestigiacomo, ${ }^{27}$ Athos Patsalides, ${ }^{28}$ Peter Rasmussen, ${ }^{29}$ Robert M Starke, ${ }^{30}$ \\ Peter Sunenshine, ${ }^{31}$ Donald Frei, ${ }^{32}$ Mahesh V Jayaraman, ${ }^{33}$ on behalf of the \\ Standards and Guidelines Committee of the Society of Neurolnterventional Surgery (SNIS)
}

For numbered affiliations see end of article.

\section{Correspondence to}

Dr Mahesh V Jayaraman, Warren Alpert Medical School of Brown University, 593 Eddy St, Room 377, Providence, RI 2903, USA; MJayaraman@Lifespan.org

Accepted 16 August 2016 Published Online First 5 October 2016

\section{CrossMark}

To cite: Pride GL, Fraser JF, Gupta R, et al.

J Neurolntervent Surg

2017:9:801-811.

\section{INTRODUCTION}

Recent randomized clinical trials ${ }^{1-5}$ established the superiority of endovascular recanalization techniques, specifically mechanical embolectomy, compared with best medical therapy alone for the treatment of patients with emergent large vessel occlusion (ELVO) stroke. ELVO stroke is defined as a stroke secondary to anterior circulation large vessel occlusion (LVO) of the internal carotid, middle cerebral (M1 segments) arteries documented by imaging, without large completed infarct and presenting within 6 hours of symptom onset. ${ }^{6}$ Given the overwhelming clinical evidence provided by these trials, recent American Heart Association (AHA) guidelines concluded that "embolectomy needs to be performed as rapidly as possible for the greatest clinical benefit, and is best when performed within $6 \mathrm{~h}$ from onset of symptoms" (AHA class I, level of evidence A). ${ }^{6}$ In addition, cost modeling derived from trial outcomes data and claims databases in the USA strongly suggests that costeffectiveness and an overall societal benefit is associated with investment in access to these endovascular techniques. ${ }^{7}$ Rapid access to endovascular services depends upon optimization of prehospital stroke care and transport within stroke systems of care, focusing on the unique needs of patients with ELVO through their diagnostic investigation and treatment pathway. The Society of NeuroInterventional Surgery (SNIS) proposed process time metrics for ELVO stroke treatment, including door to IV tissue plasminogen activator (t-PA) of $<30 \mathrm{~min}$, comprehensive stroke center (CSC) door to puncture of $<60 \mathrm{~min}, \mathrm{CSC}$ door to recanalization of $<90 \mathrm{~min}$ and primary stroke certification (PSC) picture to CSC puncture of $<90$ min. $^{8}$ Early team awareness of the patient with potential ELVO, coupled with efficient interdisciplinary communication, triage and transport assist in meeting these ideal time metrics, and also contribute to improved clinical outcomes through efficiency gains and maximization of endovascular care delivery.

\section{MATERIALS AND METHODS}

The Standards and Guidelines Committee of the SNIS, a multidisciplinary society representing leaders in the field of endovascular therapy for neurovascular disease, prepared this document based upon a comprehensive review of English language literature relating to the topic. The strength of evidence supporting each recommendation was summarized using levels of evidence as defined by the AHA.

\section{OUTCOMES AND TIME TO \\ REVASCULARIZATION IN ELVO STROKE}

The dependence of good clinical outcome on time to revascularization in ELVO stroke is well established. ${ }^{9}{ }^{10}$ Reperfusion therapy is effective at preserving penumbral tissue, the volume of which diminishes with time. ${ }^{11}$ Data from the endovascular cohort in the Interventional Management of Stroke (IMS) III trial demonstrated that shorter time to reperfusion was associated with improved outcome (modified Rankin Scale score $\leq 2$ at 90 days). ${ }^{10} \mathrm{~A}$ relative risk reduction of a good outcome of approximately $12-15 \%$ was associated with each 30-min delay in time to reperfusion (unadjusted RR with 30-min delay: $0.85,95 \%$ CI 0.77 to 0.94). Dramatic recovery in ELVO, defined as National Institutes of Health Stroke Scale (NIHSS) score $\leq 3$ at 24 hours, or a decrease of the NIHSS score of $\geq 10$ points in 24 hours, was shown to be a powerful predictor of excellent outcome (OR of modified Rankin Scale score $\leq 1$ at 90 days: 23.82, $95 \%$ CI 10.85 to $53.25 ; \mathrm{p}<0.001),{ }^{12}$ and was also independently associated with time to recanalization, being significantly more likely with each 30 -min reduction in time (OR of dramatic recovery: $1.24,95 \%$ CI 1.04 to $1.48 ; \mathrm{p}=0.016) .{ }^{12}$ 
The SPEED study, which evaluated a newer technique/catheter against older data from the PIVOTAL study ${ }^{13}$ suggests that faster reperfusion times improve outcome. ${ }^{14} \mathrm{~A}$ smaller study of patients who underwent rapid imaging to reperfusion with embolectomy found that faster treatment led to high rates of excellent clinical outcomes ( $82 \%$ of patients). ${ }^{15}$ Evaluation of the databases from the SWIFT and STAR trials determining mechanical embolectomy with stent retrievers, focusing on 202 patients in whom embolectomy was technically successful, showed that shorter times from symptom onset to recanalization were associated with significantly improved 90 -day clinical outcomes. ${ }^{16}$ The authors noted that, for every 15 -min reduction in time from onset to recanalization, 34 of every 1000 treated patients had an improved disability outcome. ${ }^{16}$

The recently published randomized controlled trials mentioned above provide further confirmation of the benefit of time (table 1). The MR CLEAN trial, ${ }^{1}$ compared with IMS III, ${ }^{17}$ which previously showed no significant added benefits of endovascular management to standard medical therapy, demonstrated clinical benefits of endovascular treatment with mechanical embolectomy without initiating an improvement in time to treatment, suggesting that the benefits shown in the study were probably related to better patient selection using imaging confirmation of LVO and better revascularization techniques employing modern devices in the endovascular group. ${ }^{18}$ Subgroup analyses from the MR CLEAN trial presented at the International Stroke Conference 2015 showed an approximately $7 \%$ decreased probability of good outcome for each hour delay in revascularization. ${ }^{19}$ For patients revascularized within 2 hours of stroke onset, the absolute difference in good outcomes between the endovascular and control groups was 33\%, decreasing to $6.5 \%$ at 6 hours. ${ }^{19}$

Subsequently published trials, with similar design to MR CLEAN but faster treatment times, also suggest a widening absolute treatment effect difference between the control and treatment groups with decreasing time to endovascular treatment (table 1). In two trials, particular emphasis was placed upon efficiency with monitored time goals of qualifying imaging to groin of $70 \mathrm{~min}$ in SWIFT PRIME ${ }^{4}$ and goals of CT to groin and CT to reperfusion of 60 and $90 \mathrm{~min}$, respectively, in ESCAPE. ${ }^{3}$ Analyses of efficiency data from these trials show clear benefits of reduced time to reperfusion, with $8.3 \%$ reduction in good outcome for every 30-min delay from imaging to reperfusion in $\mathrm{ESCAPE}^{20}$ and a $91 \%$ probability of good outcome for those in SWIFT PRIME reperfused within $150 \mathrm{~min}$ of symptom onset, decreasing by $10 \%$ for an initial hour of delay and $20 \%$ for each subsequent hour of delay. ${ }^{21}$

Table 1 Endovascular stroke trials and treatment time

\begin{tabular}{llllll}
\hline & $\begin{array}{l}\text { Time to } \\
\text { groin } \\
\text { (median) } \\
(\mathbf{m i n})\end{array}$ & $\begin{array}{l}\text { Time to } \\
\text { reperfusion } \\
\text { (median) } \\
\text { (min) }\end{array}$ & $\begin{array}{l}\text { mRS 0-2 } \\
\text { Endovascular } \\
(\%)\end{array}$ & $\begin{array}{l}\text { mRS } \\
\mathbf{0}-2 \\
\text { Medical } \\
(\%)\end{array}$ & $\begin{array}{l}\text { Absolute } \\
\text { difference } \\
(\%)\end{array}$ \\
\hline Study & 208 & 325 & 40.8 & 38.7 & 2.1 \\
\hline IMS III & 208 & 332 & 32.6 & 19.1 & 13.5 \\
MR CLEAN & 260 & 355 & 43.7 & 28.2 & 15.5 \\
REVASCAT & 269 & 355 & 60.2 & 35.5 & 24.7 \\
SWIFT & 224 & 252 & & & \\
PRIME & & & 71 & 40 & 31 \\
EXTEND IA & 210 & 248 & 53 & 29.3 & 23.7 \\
ESCAPE & 185 & 241 & 53
\end{tabular}

IMS, Interventional Management of Stroke; mRS, modified Rankin Scale.
These studies provide models for efficiency that will probably strongly influence future recommended time metrics for ELVO stroke. Clearly, time to reperfusion has a profound effect on outcome.

\section{TIMING AND EFFICIENCY IN ELVO STROKE}

Most of the time-related outcome gains realized in recently reported studies result from improvements in techniques and hospital processes involving the treatment link along the American Stroke Association (ASA)-defined 'stroke chain of survival' connecting recognition, dispatch, transport, and treatment (http://www.strokeassociation.org). Since time until treatment is a continuous variable with a non-linear effect on outcome, time savings achieved along each earlier link of recognition, dispatch, and transport may likewise significantly affect outcome in ELVO stroke. Analysis of pooled data from embolectomy trials including the multi-MERCI, TREVO, and TREVO-2 trials involving 1248 patients over a 10 -year period showed that significant improvement occurred in procedure times, without corresponding improvements in last known normal (LKN) to puncture times. Prolonged LKN to puncture times were significantly associated with a decreased chance of good outcome $(\mathrm{OR}=0.84$, $95 \%$ CI 0.76 to $0.92 ; \mathrm{p}=0.0004) .{ }^{22}$ Thus, rapid recognition and triage of patients with stroke has become paramount.

Liebeskind $e a^{23}$ analyzed data from the SWIFT trial (which compared different techniques in embolectomy for stroke) and found that a time $>3$ hours was the only predictor of extensive infarct on imaging $(p=0.003)$. In that study, shorter times from symptom onset to hospital arrival were associated with smaller infarcts, better collateral vessels, and improved clinical outcome from embolectomy. ${ }^{23}$ To that end, the AHA/ASA 2015 guidelines recently found that "Patients should be transported rapidly to the closest available certified primary stroke center or comprehensive stroke center or, if no such centers exist, the most appropriate institution that provides emergency stroke care"24 A variety of legislative efforts also support this approach. Evaluation of the endovascular cohort in the IMS III trial showed that transfer of patients between centers resulted in longer stroke onset to reperfusion times than for those treated at the same center, ${ }^{25}$ providing impetus for consideration of primary transport to endovascular-capable centers. Indeed, although local systems of care may vary, the importance of rapid recognition of diagnosis and transport to appropriate centers is consistently paramount.

\section{STROKE SYSTEMS OF CARE AND ELVO STROKE}

Systems of care for stroke are rapidly gaining importance in acute stroke intervention. As the pivotal roles of early recognition and rapid treatment have become clear, protocols for care and real-time data collection are now vital. In the 1990s and early 2000s, several organizations and healthcare bodies recognized the uneven distribution of stroke care, and the need to standardize approaches. ${ }^{26}$ Born from the efforts of the Brain Attack Coalition (BAC) with certification offered through the Joint Commission on Accreditation of Healthcare Organizations (JCAHO), PSC began in 2004. ${ }^{27}$ Later partnership with the AHA/ASA led to widespread adoption and by 2011, there were over 800 PSC hospitals. ${ }^{26}$

The maintenance of PSC requires practice of stroke care to standardized guidelines, and reporting of metrics (core measures) as a marker of success. For example, primary stroke centers must report their door-to-needle time that marks the average time interval from the moment a patient with stroke enters the hospital to the administration of IV t-PA. Primary 
stroke centers are also involved in developing stroke networks, providing education to providers, and guiding emergency medical services (EMS) protocols for stroke recognition and care. Such certification carries some benefits-improving adherence to standards of care in thrombolysis for ischemic stroke, and reducing overall mortality. ${ }^{28-30}$

However, recognizing that some hospitals were needed that could reliably offer more complex stroke care, the Joint Commission launched certification for CSCs in 2012. Although somewhat controversial, these centers, with reliable 24/7 availability of endovascular services, are ideal destinations for patients with ELVO stroke. The new certification process aligns with earlier recommendations from the BAC in 2005 for advanced centers. ${ }^{31}$ CSCs must meet requirements for PSC, but also have a minimum case volume of patients with complex ischemic and hemorrhagic stroke. Furthermore, they must have cerebrovascular imaging capabilities on site, 24/7 neurosurgical services, and a dedicated intensive care service that manages patients with complex stroke. They must also have peer-review mechanisms to discuss complex cases, participate in strokerelated research, and report additional core measures above those required by primary stroke centers. They must also be staffed sufficiently to be able to care for simultaneous complex stroke patients. $^{32}$

Early data suggest that care in a CSC is associated with improved survival at 90 days for patients with hemorrhagic stroke, who typically require more complex care. ${ }^{33} \mathrm{~A}$ review of patients with hemorrhagic stroke in the Myocardial Infarction Data Acquisition System (MIDAS) database, ${ }^{33}$ demonstrated that patients admitted to CSCs were more likely to have neurosurgical or endovascular interventions than those admitted to a primary stroke center/non-stroke center $(18.9 \%$ vs $4.7 \%$; $\mathrm{p}<0.0001)$. Furthermore, CSC admission was associated with lower adjusted 90 -day mortality $(35.0 \%$ vs $40.3 \%$; $0.93 ; 95 \%$ CI -0.89 to 0.97 ). Thus, appropriate transfer of patients with suspected or confirmed complex stroke (such as hemorrhagic stroke or ELVO) to a CSC is important within a stroke system of care.

Three levels of certification are defined by JCAHO and the AHA/ASA: acute stroke ready hospitals, primary stroke centers, and CSCs. ${ }^{32}$ Certification of centers in the USA occurs through JCAHO, and other CMS authorized certification organizations such as Det Norske Veritas/Germanischer Lloyd (DNV-GL) and the Healthcare Facilities Accreditation Program (HFAP). Additionally, individual approval of stroke centers has been organized at state levels, including through the Agency for Health Care Administration (AHCA) in Florida and the Department of State Health Services (DSHS) in Texas. Although there are some subtle differences in the requirements of these different organizations, they all adhere to the structure outlined by JCAHO, the AHA/ASA, and the BAC.

Through a 'hub and spoke' model, ${ }^{34} 35$ with the CSC serving as the hub, stroke networks can recognize and treat patients with stroke quickly, while diverting patients with complex, severe stroke to the most appropriate hospital. Ultimately, the CSCs are responsible for reporting complex metrics for all avenues of stroke treatment, including such measures as time to endovascular therapy and appropriate reversal of anticoagulation in hemorrhagic stroke. ${ }^{36}$ Certification of centers provides reliable verification of effective comprehensive services for planners of stroke systems of care at local, state, and national levels. With these levels of certification, the routing of patients by EMS becomes crucial to stroke systems of care. Indeed, routing protocols for patients with stroke can help concentrate them quickly in equipped and certified hospitals, ${ }^{37}$ enabling them to receive the optimized level of care. Several opportunities exist for interaction between EMS and stroke systems of care, which could improve efficiency and, potentially, improve outcomes for patients with ELVO stroke.

\section{PREHOSPITAL EFFICIENCY OPPORTUNITIES IN ELVO STROKE}

Prehospital efficiency improvement should focus on the two treatments with proven benefit for the patient with ELVO stroke - IV t-PA and endovascular therapy. IV t-PA should be delivered as quickly as possible to eligible patients. Randomized controlled trials have proved it to be efficacious, ${ }^{38}{ }^{39}$ and safe to administer in the setting of potential endovascular treatment. ${ }^{1-5} 17$ A significant majority of the patients treated endovascularly in SWIFT PRIME, MR CLEAN, EXTEND-IA, ESCAPE and REVASCAT received IV-t-PA before, or at the same time as, endovascular treatment $\left(459 / 634\right.$ (72.4\%) patients). ${ }^{1-5}$ Rapid access to endovascular treatment should be a goal for all patients suspected of having, or proved with imaging to have, ELVO.

\section{Public education}

Two aspects of public education are of particular importance for the patient with ELVO. First, efforts to raise awareness of ELVO stroke as a distinct subtype of stroke requiring treatment at comprehensive centers with endovascular capability may prove important. Considerable success in educating the public about stroke symptoms was achieved with the introduction in the UK of the Face/Arm/Speech Time (FAST) scale ${ }^{40}$ first to EMS and primary caregivers, later to the public. This scale was derived from earlier stroke recognition scales developed and validated in the 1990s and early 2000s-namely, the Cincinnati Prehospital Stroke Scale (CPSS), ${ }^{41}$ and the Los Angeles Prehospital Stroke Screen (LAPSS). ${ }^{42}{ }^{43}$ FAST correctly identified stroke symptoms in $88.9 \%$ of patients in one study. ${ }^{44}$ Its simplicity promotes retention, with studies showing significantly increased awareness of stroke symptoms, ${ }^{45-47}$ and objective improvement in delays in seeking and receiving treatment. ${ }^{48}$ Similar education of the public about ELVO stroke could be carried out, providing recognition tools, and a comprehensive center with endovascular services as the appropriate treatment endpoint. Although the educational tools have yet to be defined, the accumulated evidence supporting improved outcomes with endovascular treatment suggests the importance of separately identifying ELVO stroke with its increased complexity of its treatment.

Second, as with stroke in general, public awareness about the benefits of EMS transport should be raised, with patients, families and the public being encouraged to use the 911 response system instead of self-transport for suspected ELVO stroke. Rapid transport to highly specialized centers, such as primary stroke centers or CSCs, is greatly facilitated by EMS networks. In one study involving 158 hospitals in northwestern Germany, EMS transport was independently associated with faster times of hospital arrival, shorter times to brain imaging, and higher probability of treatment with thrombolysis than self-transport. ${ }^{49}$ It was estimated, based upon 2011 US demographic data and Medicare endovascular treatment rates, that $56 \%$ and $85 \%$ of the US population had ground or air access, respectively, to endovascular-capable hospitals within $60 \mathrm{~min}^{50}{ }^{5}$ When the time interval was extended to $120 \mathrm{~min}, 99 \%$ of the US population had such access. Maximization of timely access requires sustained coordination of public and EMS educational efforts about ELVO stroke, organization of EMS and stroke care networks, and ongoing assessment of process improvement. 


\section{EMS initial contact}

On initial contact with the EMS, important information, which might affect the efficiency of treatment, should be obtained and recorded. A family or next of kin contact is critically important for history gathering or obtaining treatment consent. Preferably, this should be a consistent contact number, such as a cell phone number. Many treatment decisions related to acute stroke depend upon an accurate time of onset or LKN time, and this knowledge is critical in evaluation of the patient with ELVO stroke. Agreement between EMS-determined time of onset and hospital neurologist LKN times has been shown when personnel are appropriately trained. ${ }^{51}$ Factors associated with incongruence were older patient age and wake-up strokes. A history of anticoagulant use should be obtained, as this information is useful in determining eligibility for IV t-PA.

\section{Early identification}

Strategies for streamlining stroke care include the use of EMS field screening tools that can be administered quickly and easily by EMS personnel. Most tools employed by EMS are designed for stroke recognition rather than stroke classification or severity assessment. These recognition scales include the CPSS, FAST, LAPSS, Melbourne Ambulance Stroke Screen (MASS), ${ }^{52}$ Medic Prehospital Assessment for Code Stroke (Med PACS), ${ }^{53}$ and Recognition of Stroke in the Emergency Room (ROSIER) scale. ${ }^{54}$ Tools used vary by region, but employment of some tool for stroke recognition is recommended in clinical guidelines. ${ }^{55}$

In North Carolina, retrospective comparison of hospitals with EMS databases for the diagnosis of stroke and the accuracy of CPSS and LAPSS stroke recognition tools in large numbers of patients (2442) showed sensitivity of $80 \%$ and $74 \%$, respectively, but with relatively low specificity of $48 \%$ for both scales. ${ }^{56}$ This low specificity was corroborated by another study from Michigan, ${ }^{57}$ where EMS screening had a false-positive rate of nearly $50 \%$. Factors in that study associated with increased accuracy of EMS stroke diagnosis included documentation of administration of the CPSS, higher NIHSS (more severe strokes; $\mathrm{OR}=1.09$ for each one point increase), and early presentation $(\mathrm{OR}=2.22)$. Another study showed an overall low sensitivity of $62.4 \%$ for EMS recognition of stroke, but no patients with strokes presenting with unilateral weakness, facial weakness, or speech problems were missed. ${ }^{58}$ Despite the relatively low overall specificity and sensitivity in these studies, these associations support use of prehospital screening scales to identify patients with stroke and suggest that EMS accuracy is higher for patients with severe strokes at early time intervals, exactly the population most likely to have LVOs that might benefit from endovascular treatment.

Early identification of patients with suspected ELVO stroke can focus the entire team on facilitating delivery of endovascular care at a CSC as early as possible either through direct transport or through later interfacility transfer. EMS can be instrumental in this regard, administering recognition tools to direct appropriate triage. ${ }^{59}$ Standardization and evaluation of field clinical assessment tools for ELVO stroke have been in development for several years, with several tools described in the literature ${ }^{60-63}$ sharing some common themes. Higher stroke severity and the presence of cortical signs have been associated with a higher likelihood of LVO in patients with acute stroke undergoing vascular imaging with angiography, MR angiography (MRA), or CT angiography (CTA). ${ }^{64}{ }^{65}$ NIHSS scores $\geq 12$ were associated with a 91\% positive predictive value (PPV) for central LVO. ${ }^{64} \mathrm{~A}$ degree of time dependence was also demonstrated, with a NIHSS score $\geq 9$ associated with a PPV for LVO of $86.4 \%$ within 3 hours of onset, but a lower NIHSS score $\geq 7$ associated with a similar PPV for LVO of $84.4 \%$ between 3 and 6 hours of stroke onset, ${ }^{65}$ so absolute NIHSS score cut-off points are problematic. Additional independent associations with LVO included motor function of the leg and portions of the NIHSS concerned with assessment of cortical functions, including gaze, level of consciousness questions, and neglect. ${ }^{64}$ The lengthy NIHSS is not well suited to application in the field by EMS, compelling the development of simpler scales that can be more rapidly evaluated. Severe hemiparesis or hemiplegia was associated with a cerebrovascular etiology in $84.5 \%$ of 45 patients transported to a single CSC by helicopter over a period of 6 months, including $60 \%$ ischemic stroke, $13.3 \%$ intracerebral hemorrhage, $15.5 \%$ transient ischemic attack, and 2.2\% subarachnoid hemorrhage. ${ }^{66}$ Of the transported patients, $26.7 \%$ underwent mechanical thrombectomy for ELVO and 33\% underwent an endovascular procedure for either ischemic or hemorrhagic stroke, suggesting that this clinical finding alone may indicate the potential for improved outcomes by transport to facilities with endovascular services.

Stroke field severity scales associated with a high probability of ELVO, either directly or through association with high NIHSS scores, include the 3-Item Stroke Scale (3I-SS), ${ }^{60}$ the Los Angeles Motor Scale (LAMS), ${ }^{61}$ the Rapid Arterial oCclusion Evaluation (RACE) scale, ${ }^{63}$ the Cincinnati Prehospital Stroke Severity Scale (CPSSS), ${ }^{62}$ the LEGS score, ${ }^{67}$ the VAN (vision, aphasia, neglect) screening tool, ${ }^{68}$ and several shortened variations of the NIHSS. ${ }^{69} 70$

The 3I-SS is a simple scale derived from the NIHSS evaluating gaze and head deviation, level of consciousness, and motor function. ${ }^{60}$ It is strongly associated with the NIHSS for assessing stroke severity, and correlated with ELVO in prospectively evaluated patients undergoing MRA, with a high degree of interobserver reliability (intraclass correlation coefficient 0.947 ). The optimal level of severity to predict ELVO was a score $\geq 4$, with an overall accuracy of 0.86 (table 2). More intracranial hemorrhages were associated with both a higher 3I-SS and NIHSS scores.

The LAMS incorporates three motor components of the LAPSS stroke recognition scale and is relatively quickly administered, taking about $20-30 \mathrm{~s},{ }^{61}$ concurrent with the LAPSS. At

Table 2 The 3-Item Stroke Scale (3I-SS) ${ }^{60}$

\begin{tabular}{lr}
\hline Item & Points \\
\hline Consciousness disturbance & \\
None & 0 \\
Mild & 1 \\
Severe & 2 \\
Gaze and head deviation & \\
Absent & 0 \\
Incomplete & 1 \\
Complete & 2 \\
Hemiparesis & \\
Absent & 0 \\
Moderate & 1 \\
$\quad$ Severe & 2 \\
Total score & $0-6$ \\
\hline 3l-SS $\geq 4: 67 \%$ sensitivity, 92\% specificity, 0.36 negative likelihood ratio for large \\
vessel occlusion.
\end{tabular}

Pride GL, et al. J Neurolntervent Surg 2017;9:802-812. doi:10.1136/neurintsurg-2016-012699 
the optimal threshold of $\geq 4$ points, this scale showed an overall accuracy of 0.85 for the presence of ELVO in retrospective score derivations from trial and registry anterior circulation stroke databases (table 3). Although the LAMS does not incorporate an assessment of cortical function, a sevenfold increased incidence of ELVO was found with LAMS $\geq 4$ points.

The RACE scale likewise incorporates motor function, but adds assessment of cortical function of each hemisphere, with aphasia assessment for the left hemisphere and agnosia assessment for the right hemisphere. ${ }^{63}$ It was derived from retrospective analysis of a large Spanish stroke cohort to identify portions of the NIHSS most highly correlated with ELVO. Prospective validation in the field by EMS yielded an accuracy for prediction of ELVO of 0.72 at the optimal score of $\geq 5$ points (table 4$){ }^{63}$ Higher RACE scores correlated strongly with the presence of ELVO and of hemorrhagic stroke and correlated less well with stroke mimics.

The CPSSS is a relatively simple scale incorporating gaze, level of consciousness, and motor components of the NIHSS. ${ }^{62}$ These were derived using classification and regression tree analysis of NINDS t-PA trial data to identify NIHSS components that correlated best with NIHSS stroke severity $\geq 15$. Advantages associated with this scale include its brevity, use of less subjective dichotomous responses, and incorporation of gaze abnormalities. CPSSS scores of $\geq 2$ points were associated with an accuracy of 0.89 for the detection of severe stroke with a NIHSS score $\geq 15$. Validation with the IMS III dataset for the presence of ELVO on CTA yielded an accuracy of 0.67 for CPSSS scale scores $\geq 2$ points (table 5 ).

The VAN screening tool incorporates assessment of vision, aphasia, and neglect with motor assessment of arm strength. ${ }^{68} \mathrm{It}$ is a dichotomous tool rather than a numeric scale with the overall screen considered positive for weakness combined with any positive vision, aphasia, or neglect screen. Prospective single-center correlation with ELVO when administered by trained emergency room nurses yielded an accuracy of 0.92 (table 6).

From the above discussion, it is clear that several similar prehospital stroke severity/ELVO identification scales are available with similar reported accuracy. Scales such as the 3I-SS, CPSSS, LAMS, and the VAN screening tool have the advantage of being simple and easy to administer by EMS, but have not yet undergone prospective validation. The RACE scale, although more complicated to administer, has undergone prospective field EMS

Table 3 Los Angeles Motor Scale (LAMS) ${ }^{61}$

\begin{tabular}{lr}
\hline Item & Points \\
\hline Facial droop & 0 \\
Absent & 1 \\
Present & \\
Arm drift & 0 \\
Absent & 1 \\
Drifts down & 2 \\
Falls rapidly & \\
Grip strength & 0 \\
Normal & 1 \\
Weak grip & 2 \\
No grip & $0-5$ \\
Total score & \\
\hline LAMS $\geq 4: 81 \%$ sensitivity, 89\% specificity, 0.21 negative likelihood ratio for large \\
vessel occlusion.
\end{tabular}

validation. Given the overwhelming data supporting endovascular treatment, further studies of these tools in the field are needed. There is insufficient evidence to state which scale or field tool is optimal, but we recommend that EMS use one of them to identify patients with ELVO in the field. Direct transport to comprehensive centers with endovascular capability for severe strokes with a high probability of ELVO maximizes time savings for potential endovascular treatment, and ensures that appropriate neurosurgical and neurointensive care is readily available for those patients with hemorrhagic strokes and severe strokes identified by higher scores on these scales. Indeed, patients with hemorrhagic strokes treated at CSCs have improved survival. ${ }^{33}$

Perhaps the most accurate means of identifying a patient with ELVO requires imaging equipment or other testing being brought to the patient. Transcranial ultrasound has been used in the prehospital setting with adequate personnel and training. ${ }^{71}$ 72-74 This modality can diagnose middle cerebral artery (MCA) occlusion with a reasonably high degree of accuracy. In one German study, transcranial ultrasound used in a prehospital setting demonstrated a sensitivity of $78 \%$ and specificity of $98 \%$ for the diagnosis of MCA or internal carotid artery occlusion in comparison with CTA/MRA performed at receiving hospitals. ${ }^{74}$ In addition, investigations are continuing into the use of ultrasound, with or without microbubble contrast administration, to assist in mechanical clot dissolution or aid the cerebral microcirculation, potentially providing some degree of prehospital clot 'conditioning' or neuroprotection before definitive treatment. ${ }^{73}$ An advantage of transcranial ultrasound includes its portability,

Table 4 Rapid Arterial oCclusion Evaluation scale (RACE) ${ }^{63}$

\begin{tabular}{|c|c|c|}
\hline Item & $\begin{array}{l}\text { Race } \\
\text { score }\end{array}$ & $\begin{array}{l}\text { NIHSS } \\
\text { equivalent }\end{array}$ \\
\hline \multicolumn{3}{|l|}{ Facial palsy } \\
\hline Absent & 0 & 0 \\
\hline Mild & 1 & 1 \\
\hline Moderate to severe & 2 & $2-3$ \\
\hline \multicolumn{3}{|l|}{ Arm motor function } \\
\hline Normal to mild & 0 & $0-1$ \\
\hline Moderate & 1 & 2 \\
\hline Severe & 2 & $3-4$ \\
\hline \multicolumn{3}{|l|}{ Leg motor function } \\
\hline Normal to mild & 0 & $0-1$ \\
\hline Moderate & 1 & 2 \\
\hline Severe & 2 & $3-4$ \\
\hline \multicolumn{3}{|l|}{ Head and gaze deviation } \\
\hline Absent & 0 & 0 \\
\hline Present & 1 & $1-2$ \\
\hline \multicolumn{3}{|l|}{ Aphasia* (if R hemiparesis) } \\
\hline Performs both tasks correctly & 0 & 0 \\
\hline Performs one task correctly & 1 & 1 \\
\hline Performs neither task & 2 & 2 \\
\hline \multicolumn{3}{|l|}{ Agnosiat (if L hemiparesis) } \\
\hline Recognizes both paretic arm and impairment & 0 & 0 \\
\hline Does not recognize paretic arm or impairment & 1 & 1 \\
\hline Does not recognize paretic arm and impairment & 2 & 2 \\
\hline Total score & $0-9$ & \\
\hline
\end{tabular}

*Aphasia: (1) 'close your eyes'; (2) 'make a fist'.

†Agnosia: (1) "'whose arm is this?'"; (2) 'raise both hands and clap'.

RACE $\geq 5: 85 \%$ sensitivity, $68 \%$ specificity, 0.22 negative likelihood ratio for large vessel occlusion.

NIHSS, National Institutes of Health Stroke Scale. 
Table 5 Cincinnati Prehospital Stroke Severity Scale (CPSSS) ${ }^{62}$

\begin{tabular}{|c|c|}
\hline Item & Points \\
\hline \multicolumn{2}{|l|}{ Gaze } \\
\hline Conjugate gaze deviation ( $\geq 1$ NIHSS gaze) & 2 \\
\hline \multicolumn{2}{|l|}{ Consciousness/commands } \\
\hline $\begin{array}{l}\text { Incorrectly answers one LOC question and one command on NIHSS } \\
\text { (age, current month, close eyes, open and close hand) ( } \geq 1 \text { on } \\
\text { NIHSS) }\end{array}$ & 1 \\
\hline \multicolumn{2}{|l|}{ Motor arm } \\
\hline $\begin{array}{l}\text { Cannot hold arm up (left, right or both) for } 10 \mathrm{~s} \text { before it falls to bed } \\
\text { ( } \geq 2 \text { on NIHSS) }\end{array}$ & 1 \\
\hline Total score & $0-4$ \\
\hline \multicolumn{2}{|l|}{$\begin{array}{l}\text { CPSSS } \geq 2: 83 \% \text { sensitivity, } 40 \% \text { specificity, } 0.4 \text { negative likelihood ratio for large } \\
\text { vessel occlusion. } \\
\text { CPSSS } \geq 2: 92 \% \text { sensitivity, } 51 \% \text { specificity, } 0.15 \text { negative likelihood ratio for NIHSS } \\
\text { score } \geq 15 \text {. } \\
\text { LOC, level of consciousness; NIHSS, National Institutes of Health Stroke Scale. }\end{array}$} \\
\hline
\end{tabular}

\begin{tabular}{|c|c|}
\hline Item & Responses \\
\hline \multicolumn{2}{|l|}{ Motor arm } \\
\hline Raise both arms & $\begin{array}{l}\text { Mild-minor drift } \\
\text { Moderate-severe drift } \\
\text { Severe-flaccid or no antigravity } \\
\text { No weakness-VAN negative }\end{array}$ \\
\hline \multicolumn{2}{|l|}{ Vision } \\
\hline $\begin{array}{l}\text { Assessment of visual fields, } \\
\text { vision, diplopia }\end{array}$ & $\begin{array}{l}\text { Field cut } \\
\text { Double vision } \\
\text { New blindness } \\
\text { None }\end{array}$ \\
\hline \multicolumn{2}{|l|}{ Aphasia } \\
\hline $\begin{array}{l}\text { Repeat and name two } \\
\text { objects } \\
\text { Open and close eyes and fist }\end{array}$ & $\begin{array}{l}\text { Expressive } \\
\text { Receptive } \\
\text { Mixed } \\
\text { None }\end{array}$ \\
\hline \multicolumn{2}{|l|}{ Neglect } \\
\hline $\begin{array}{l}\text { Gaze preference, tactile or } \\
\text { spatial neglect }\end{array}$ & $\begin{array}{l}\text { Forced gaze or inability to track to one side } \\
\text { Unable to feel both sides at the same time or } \\
\text { unable to identify own arm } \\
\text { Ignores one side } \\
\text { None }\end{array}$ \\
\hline
\end{tabular}

allowing application in air- and ground-based transport. ${ }^{73}$ Disadvantages include a requirement for intensive personnel training, with direct physician supervision either physically or electronically, and the limitations imposed by cranial bony imaging windows and the inability to diagnose hemorrhage.

Improvements in diagnosis and treatment have been achieved through mobile stroke evaluation/treatment units (STEMO (stroke emergency mobile), MSU (mobile stroke unit), MSTU (mobile stroke treatment unit) $)^{75}$ incorporating portable CT scanners, point of care testing, medication delivery, and direct or remote neurological expertise in a customized ambulance, first in Germany, ${ }^{76}$ subsequently in several centers in the USA. $^{77-79}$ This enables accurate diagnosis in the field, and medical therapy to be delivered to higher percentages of patients at earlier times. In the German experience, higher rates of IV t-PA administration ( $32 \%$ vs $22 \%, \mathrm{p}<0.001)$, higher treatment rates within 1 hour of symptom onset (31\% vs 4.9\%, $\mathrm{p}<0.01)$, and an improved likelihood of treated patients being discharged home (adjusted $\mathrm{OR}=1.93, \mathrm{p}=0.02$ ) were achieved with the use of STEMO compared with standard, conventional care ${ }^{80}$ Early experience with MSU in Houston demonstrated reduced times from stroke onset to groin access (average $171 \mathrm{~min}$ ) compared with published time metrics in patients receiving endovascular care after MSTU transport. ${ }^{78}$ A similar experience in Cleveland showed substantial reduction in time metrics (door to CT $12 \mathrm{~min}$ vs $32 \mathrm{~min}$, CT to IA therapy $82 \mathrm{~min}$ vs $165 \mathrm{~min}$ ) for MSTU-transported patients undergoing ultimate endovascular treatment compared with those who underwent such treatment after standard EMS transport. ${ }^{81}$ These efficiency gains highlight the time savings associated with MSTU evaluation/treatment, which should translate to improved outcomes. Economic evaluation suggested that MSUs are costefficient when used in an operating distance of greater than 9.99 miles and a population density of 202 inhabitants per square mile or greater. ${ }^{82}$ Whether the promise of these mobile units will be fulfilled on a larger scale remains to be seen.

\section{Prehospital notification}

Prehospital notification of transport is an important efficiency improvement strategy. EMS transport and prehospital notification of patients with suspected stroke reduces stroke time metrics and increases the proportion of patients receiving IV $\mathrm{t}-\mathrm{PA},{ }^{83-87}$ but has yet to be proved to improve patient outcomes. McKinney et $a l^{84}$ found that this notification was associated with twice as many patients receiving IV t-PA. EMS transport to, or primary presentation at, stroke centers is associated with better treatment outcomes than primary presentation at community hospitals. ${ }^{88}$ Once patients with suspected ELVO have been identified, important elements to include in the notification are the LKN time, hemodynamic parameters, family contact information, and anticoagulation status. Stroke networks should integrate primary and associated comprehensive center notification to maximize transfer efficiency when transfer protocols dictate initial transport of patients with suspected ELVO to centers without endovascular services. This PSC/CSC pair integration ${ }^{8}$ would allow the comprehensive center to prepare for patient arrival and facilitate early communication between primary and comprehensive centers. The effect of prehospital notification may be magnified for ELVO stroke given the non-linear decay in treatment effect. The complex endovascular team, including radiologic technologists, neurointerventionalists, anesthesiologists, and trained nursing staff, takes some time to assemble it, particularly after hours when the team has to reach the treating center.

\section{ELVO stroke transport}

Prehospital delays can affect outcomes by causing endovascular treatment delays, and by making patients with ELVO ineligible for treatment according to the length of time or other defined clinical criteria. The treatment effect of mechanical embolectomy for patients with ELVO is substantial, with one of three to four patients benefiting in several large trials. ${ }^{2-4}$ In a Spanish study of futile transfer between facilities for endovascular therapy with a median transfer time of $60 \mathrm{~min}, 32 \%$ of the patients excluded from endovascular treatment were found to be ineligible at the receiving facility based upon the imaging progression of stroke. ${ }^{89}$ EMS transport protocols for patients with ELVO, based on best evidence, must ensure equitable population access to this effective therapy. These protocols should consider patient preference, time from symptom onset 
or LKN, overall clinical stability, transport distance, stroke severity/ELVO probability, and the capabilities of regional centers. $^{35} 90$

The primary transport requirement for the patient with suspected ELVO is prompt endovascular treatment. Ideally, the secondary transfer of patients between centers before therapy should be avoided. Protocols should only diverge from comprehensive, endovascular-capable centers for closer centers in the event of unstable patients, hypoglycemia or perhaps IV t-PA eligible patients who might miss their treatment opportunity at the closer facility by being transported directly to a more distant comprehensive center. These transport differences represent major and potentially catastrophic sources of delay for the patient with ELVO, with a median delay of $104 \mathrm{~min}$ in one urban study having a relatively short median hospital transfer distance of 14.7 miles between the primary and comprehensive centers. $^{91}$ The odds of receiving endovascular treatment were reduced in that study by $2.5 \%$ for each minute of transfer delay. In another study, ${ }^{92}$ transfer times to a single comprehensive center were consistently longer than expected driving times, highlighting these delays. Formal studies of stroke systems of care interventions relating to ELVO are being organized and should inform recommendations in an area of rapid development.

Although stroke practitioners disagree about the priority of transport, ${ }^{93}$ protocols prioritizing direct transport of patients with suspected ELVO to facilities with endovascular services are used and becoming more refined. They include bypassing closer facilities without endovascular services if transport time differences are not too great. The optimal transport time difference between centers is not firmly established, but it is generally accepted that a bypass of closer facilities should be considered if the transport time difference is $15-20 \mathrm{~min}$ for higher level stroke centers. This time has been recommended partially based upon the time required to mobilize the acute stroke team at the receiving hospital, ${ }^{35}$ particularly when no in-house team is available. This seems reasonable for IV t-PA eligible patients with suspected ELVO given the benefit afforded them by the receipt of IV t-PA as early as possible. ${ }^{94}$ Bypass of closer facilities for more capable distant facilities has been advocated for transport time differences as long as $30 \mathrm{~min}$ in cases of major trauma or ST-segment elevation myocardial infarction (STEMI). ${ }^{95} 96$ For patients with suspected ELVO who are ineligible for IV t-PA on clinical or time grounds (last known well $>3.5$ hours), strong consideration for direct transport to a comprehensive center with endovascular capability should be given, regardless of transport time differences. Implementation of statewide protocols for direct transport of patients with suspected ELVO to facilities with endovascular services is currently occurring locally. $^{97}$

Bypassing centers incapable of providing endovascular services has been evaluated for acute coronary syndromes with STEMI. In North Carolina, the RACE program encouraging bypass of non-percutaneous coronary intervention (PCI)-capable centers, even though they were closer, allowed evaluation of performance through a retrospective registry review published in 2013 in Circulation. ${ }^{98}$ They evaluated bypass of closer non-PCI capable facilities for direct transport to PCI-capable facilities and reported statistically significant reductions in time to PCI and better adherence to time guidelines for PCI in those patients taken directly to PCI centers. Trends for better outcomes were also seen, although not statistically significant after adjusting for cases of cardiac arrest. In addition, better outcomes have been demonstrated in patients with STEMI randomized to be transferred for PCI versus local thrombolysis at non-PCI capable facilities. ${ }^{99}$

For patients with suspected ELVO transported to primary facilities without endovascular services, rapid stabilization, evaluation, and transfer maximize the opportunity for effective endovascular treatment at the receiving comprehensive facility. Identification and labeling of the patient with suspected ELVO by prehospital screening may focus the entire team on time and facilitation of transfer. Although primary facilities should be able to perform vascular evaluation non-invasively to identify LVO ${ }^{8}$ this testing is not mandatory if it cannot be performed and interpreted expeditiously. Indeed, advanced imaging at the primary facility before transfer may contribute to delays (111 min, IQR 73-179, compared with $54 \mathrm{~min}$, IQR 32-76, for non-contrast CT alone; $\mathrm{p}<0.001) .{ }^{100}$ Imaging should be expeditious and directed towards excluding hemorrhage and evaluating candidacy for IV t-PA. For patients with suspected ELVO, protocols expediting evaluation may include performing imaging before formal emergency room evaluation based upon prehospital screening. Strategies to improve efficiency should involve 'drip and ship' paradigms ${ }^{101}$ to allow t-PA infusion immediately before or during transport to endovascular-capable centers; ${ }^{102}$ this requires tight coordination with EMS teams.

Time metrics for process improvement in ELVO stroke have assumed importance, many of which are correlated with outcome. Since significant delays can be introduced in the primary evaluation hospital before transfer to an endovascularcapable facility, a time metric to assess and improve delays seems helpful. For STEMI, emergency department (ED) arrival to ED discharge-'door in-door out' times ${ }^{103}$ - at the primary, non-PCI capable facility as well as 'door 1 -door 2' times ${ }^{104}$ between hospitals, have been proposed as metrics to assess the interhospital transfer processes. However, the usefulness of these measures in stroke systems of care has been questioned since $<50 \%$ of the continuum of care from patient presentation to reperfusion is accounted for by these metrics.

The 'picture to puncture' (P2P) metric assessing time from primary hospital CT to groin puncture at the recipient comprehensive center has been advanced as a better metric, encompassing about $74 \%$ of the continuum of processes involved in transfers of patients, as well as distance. ${ }^{100}$ Outside transfers were shown to have substantially longer P2P times $(205 \mathrm{~min}$, IQR 162-274 compared with 89 min, IQR 70-119; p<0.001) than those for patients with ELVO transported directly to a comprehensive facility. This longer time correlated with worse Alberta Stroke Program Early CT (ASPECT) scores on receiving hospital CT and worse clinical outcomes. The P2P time was also independently correlated with outcome $(\mathrm{OR}=0.994,95 \% \mathrm{CI}$ 0.990 to $0.999 ; \mathrm{p}=0.009) .{ }^{100}$ For every $10 \mathrm{~min}$ delay in $\mathrm{P} 2 \mathrm{P}$, there was a $6 \%$ decrease in the probability of a good outcome. A time metric proposed as a goal for P2P is <90 min. ${ }^{8} 100$

Methods of transport from the field or primary facility to comprehensive facilities differ based upon distance and rural versus urban settings. Air transport shortens transport time, ${ }^{105}$ improves survival in patients with trauma, ${ }^{106}$ and may lower the incidence of infarction, stroke, or death in patients with STEMI. ${ }^{107}$ Similar benefits to patients with stroke include providing access to IV t-PA or interventional stroke care in rural settings. ${ }^{108} 109$ Urban areas with airspace restrictions, heavy road traffic, and multiple geographically concentrated facilities create a different set of transport challenges. In this setting with an increased density of comprehensive endovascular-capable facilities, strategies requiring transport of interventional teams to multiple different facilities may be warranted (Fifi J, et al. SNIS 
presentation July 2015, San Francisco, California, USA). Regardless of the transport mechanism, EMS providers must be given tools for delineation of facility competence, such as certification status and license to transport across state lines or outside of established networks to the most appropriate facilities.

\section{Interdisciplinary communication}

Interdisciplinary communication between members of stroke networks is vitally important. Electronic communication brings expertise to the field through 'telestroke'-that is, telemedical outreach for acute stroke evaluation and management. ${ }^{110}$ Additionally, mobile technology aids can be used to facilitate communication between caregivers. Efforts should be made in the organization and delivery of care, and in the communication of outcome to remote members of the care team to stimulate team cohesion. Since efficient acute treatment of patients with ELVO stroke may involve transport outside of home communities and care networks, return home at appropriate times after treatment may facilitate recovery and foster partnerships.

\section{SUMMARY AND RECOMMENDATIONS}

1. Patients with ELVO with anterior circulation stroke secondary to occlusion of the internal carotid artery or M1 segment of the MCA and a corresponding clinical deficit benefit from efficient endovascular embolectomy. Embolectomy needs to be performed as rapidly as possible for the greatest clinical benefit, and is best when performed within 6 hours from onset of symptoms.

(AHA class I, level of evidence A)

2. EMS systems within stroke systems of care should have prehospital protocols specific to patients with a high likelihood of ELVO, including identification, transport prioritization, and efficient delivery to comprehensive centers capable of endovascular treatment to minimize treatment delays that can profoundly affect outcome.

(AHA class I, level of evidence A)

A. IDENTIFICATION. EMS systems should adopt the use of a field stroke severity scale associated with ELVO (such as 3I-SS, LAMS, RACE, CPSSS, VAN) to identify patients with suspected ELVO and prioritize transport. Further experience and study to determine the optimal field scale should continue.

(AHA class I, level of evidence B)

B. IDENTIFICATION/POINT OF CARE TREATMENT. Transcranial ultrasound is a diagnostic and therapeutic method that may identify ELVO in the field and facilitate definitive treatment through prehospital thrombus 'conditioning' or neuroprotection. This strategy will benefit from further experience and study. (AHA class II, level of evidence C)

C. IDENTIFICATION/POINT OF CARE TREATMENT. The mobile stroke unit (STEMO, MSU, MSTU) strategy reduces transport delays and times to treatment for patients with ELVO and may improve outcomes. This strategy will benefit from further experience and study. (AHA class Ii, level of evidence C)

D. TRANSPORT PRIORITIZATION. Patients with suspected ELVO based on field testing (3I-SS $\geq 4$, LAMS $\geq 4$, RACE $\geq 5$, CPSSS $\geq 2$, or VAN positive) who are eligible for IV $t$-PA (LKN $<3.5$ hours) should be considered for direct transport to a comprehensive center with endovascular treatment capability, bypassing closer facilities without this capability, if the transport difference to the closer facility is less than or equal to $15-30 \mathrm{~min}$. (AHA class I, level of evidence B)

E. TRANSPORT PRIORITIZATION. Patients with suspected ELVO based on field testing (3I-SS $\geq 4$, LAMS $\geq 4$, RACE $\geq 5$, CPSSS $\geq 2$, or VAN positive) who are ineligible for IV $t$-PA (LKN >3.5 hours) should be transported directly to a comprehensive center with endovascular treatment capability, bypassing closer facilities without this capability, if feasible.

(AHA class I, level of evidence C)

F. EFFICIENT DELIVERY. Patients with suspected ELVO primarily transported to non-endovascular-capable centers owing to large transport time differences or clinical instability should undergo expeditious evaluation and treatment, including non-contrast head CT, possible vessel imaging immediately upon arrival, and administration of IV t-PA (if eligible). Vessel imaging should not delay patient transfer. Rapid transfer to a comprehensive center with endovascular capability to minimize interhospital transfer delays is a priority. A metric proposed to assess transfer processes is the picture to puncture (P2P) time. A P2P of $<90$ min should be a goal.

(AHA class I, level of evidence C)

\section{Author affiliations}

'Department of Radiology and Neuroradiology, UT Southwestern Medical Center, Dallas, Texas, USA

${ }^{2}$ Department of Neurological Surgery, University of Kentucky, Lexington, Kentucky, USA

${ }^{3}$ Wellstar Neurosciences Institutes, Wellstar Health System, Kennestone Hospital,

Marietta, Georgia, USA

${ }^{4}$ Department of Neurology, University of Texas Southwestern Medical Center. Dallas, Texas

${ }^{5}$ Brackenridge Medical Center, Austin, USA

${ }^{6}$ Department of Emergency Medicine, University of Texas Southwestern Medical Center, Dallas, Texas, USA

${ }^{7}$ Department of Radiology, Northwestern Memorial Hospital, Chicago, Illinois, USA

${ }^{8}$ Department of Neurosurgery, University of Cincinnati, Cincinnati, Ohio, USA

${ }^{9}$ Department of Neurointerventional Surgery, Christiana Care Health Systems,

Newark, Delaware, USA

${ }^{10}$ Semmes-Murphey Neurologic and Spine Institute, Memphis, Tennessee, USA

${ }^{11}$ Department of Radiology, University of Tennessee Medical Center, Chatanooga, Tennessee, USA

${ }^{12}$ Department of Neurosurgery, Yale University School of Medicine, New Haven, Connecticut, USA

${ }^{13}$ Department of Neurosurgery, Rush University Medical Center, Chicago, Illinois, USA

${ }^{14}$ Section of Neurointerventional Radiology, Neuroscience Institute, Abbott

Northwestern Hospital, Consulting Radiologists Ltd, Minneapolis, Minnesota, USA

${ }^{15}$ Neurological Institute of New Jersey, New Jersey Medical School, Newark, New Jersey, USA

${ }^{16}$ Department of Radiology, Forsyth Medical Center, Winston Salem, North Carolina, USA

${ }^{17}$ University of Medicine, Stanford, California, USA

${ }^{18}$ Department of Interventional Neuroradiology, Massachusetts General Hospital, Boston, Massachusetts, USA

${ }^{19}$ Cerebrovascular Center, Cleveland Clinic, Cleveland, Ohio, USA

${ }^{20}$ Department of Interventional Neuroradiology, Methodist Hospital Houston,

Houston, Texas, USA

${ }^{21}$ Department of Radiology, University of Chicago, Chicago, Illinois, USA

${ }^{22}$ Department of Neurosurgery, Keck Medicine of USC, Los Angeles, California, USA

${ }^{23}$ NeuroEndovascular Program, Massachusetts General Hospital, Harvard Medical

School, Boston, Massachusetts, USA

${ }^{24}$ Department of Diagnostic Imaging, Warren Alpert School of Medicine at Brown University, Rhode Island Hospital, Providence, Rhode Island, USA

${ }^{25}$ Radiology and Neurological Surgery, Columbia University, New York, New York, USA

${ }^{26}$ Department of Neurosurgery, Icahn School of Medicine at Mount Sinai, New York, New York, USA

${ }^{27}$ Department of Neurological Surgery, New Jersey Medical School, University of Medicine and Dentistry of New Jersey, Newark, New Jersey, USA

${ }^{28}$ Department of Neurological Surgery, Weill Cornell Medical College, New York Presbyterian Hospital, New York, New York, USA 
${ }^{29}$ Neurosurgery Department, Cleveland Clinic, Cleveland, Ohio, USA

${ }^{30}$ Neurological Surgery, University of Virginia, Charlottesville, Virginia, USA

${ }^{31}$ Department of Radiology, Banner Univeristy Medical Center, Phoenix, Arizona, USA

${ }^{32}$ Interventional Neuroradiology, Radiology Imaging Associates, Englewood, Colorado, USA

${ }^{33}$ Warren Alpert School of Medical at Brown University, Providence, Rhode Island, USA

Disclaimer This literature review is provided for informational and educational purposes only. Adherence to any recommendations included in this review will not ensure successful treatment in every situation. Furthermore, the recommendations contained in this review should not be interpreted as setting a standard of care, or be deemed inclusive of all proper methods of care or exclusive of other methods of care reasonably directed to obtaining the same results. The final judgment about the propriety of any specific therapy must be made by the physician and the patient in light of all the circumstances presented by the individual patient, and the known variability and biological behavior of the medical condition. This review and its conclusions and recommendations reflect the best available information at the time it was prepared. The results of future studies may require revisions to the recommendations in this review to reflect new data. SNIS does not guarantee the accuracy or completeness of the review and assumes no responsibility for any injury or damage to people or property arising out of, or related to, any use of this review or for any errors or omissions.

Competing interests SAA: local principal investigator (PI) for HEAT, FRED and STARRT trials. JEDA: consultant for Medtronic, Penumbra, Sequent Medical and Accriva Diagnostics. DH: consultant for Stryker Neurovascular. SWH: consultant for Medina and Neuravi, and research contracts with Stryker Neurovascular, Siemens, MicroVention Terumo. Hirsch: consultant for Medtronic. RK: proctor and speaker for Medtronic. JM: consultant for Rebound Therapeutics, TSP Inc, Cerebrotech, Lazarus Effect, Pulsar, Medina;. investor in Blockade Medical, TSP Inc, Lazarus effect, Medina; PI/Co-PI for THERAPY (PI), FEAT (PI), INVEST (Co-PI), COMPASS (Co-PI), LARGE (Co-PI), COAST (Co-PI), POSITIVE (Co-PI) trials; on steering committee for the MAPS trial. CP: consultant for Codman Neurovascular (serving on Data and Safety Monitoring Board (DSMB)). GLP: consultant for Sequent Medical (DSMB for web-IT study). PR: investor, Scientific Advisory Board and stock holder—Blockade Medical; consultant/honorarium-Medtronic; Scientific Advisory Board and stock holderNervive Medical and Perflow Medical; Scientific Advisory Board—Stryker Neurovascular. PS: protctoring for Medtronic; investigator in STRATIS (Medtronic) and CARE (Penumbra) studies.

\section{REFERENCES}

1 Berkhemer OA, Fransen PSS, Beumer D, et al. A randomized trial of intraarterial treatment for acute ischemic stroke. N Engl J Med 2015;372:11-20.

2 Campbell BCV, Mitchell PJ, Kleinig TJ, et al. Endovascular therapy for ischemic stroke with perfusion-imaging selection. N Engl J Med 2015;372:1009-18.

3 Goyal M, Demchuk AM, Menon BK, et al. Randomized assessment of rapid endovascular treatment of ischemic stroke. N Engl I Med 2015;372:1019-30.

4 Saver JL, Goyal M, Bonafe A, et al. Stent-retriever thrombectomy after intravenous t-PA vs. t-PA alone in stroke. N Engl J Med 2015;372:2285-95.

5 Jovin TG, Chamorro A, Cobo E, et al. Thrombectomy within 8 hours after symptom onset in ischemic stroke. N Engl I Med 2015;372:2296-306.

6 Jayaraman MV, Hussain MS, Abruzzo T, et al. Embolectomy for stroke with emergent large vessel occlusion (ELVO): report of the Standards and Guidelines Committee of the Society of Neurolnterventional Surgery. J Neurointerv Surg 2015:7:316-21.

7 Leppert MH, Campbell JD, Simpson JR, et al. Cost-effectiveness of intra-arterial treatment as an adjunct to intravenous tissue-type plasminogen activator for acute ischemic stroke. Stroke 2015;46:1870-6.

8 McTaggart RA, Ansari SA, Goyal M, et al. Initial hospital management of patients with emergent large vessel occlusion (ELVO): report of the standards and guidelines committee of the Society of Neurolnterventional Surgery. I Neurointerv Surg Published Online First: 31 Aug 2015. doi:10.1136/neurintsurg-2015-011984

9 Khatri P, Abruzzo T, Yeatts SD, et al. Good clinical outcome after ischemic stroke with successful revascularization is time-dependent. Neurology 2009;73:1066-72.

10 Khatri $\mathrm{P}$, Yeatts SD, Mazighi M, et al. Time to angiographic reperfusion and clinical outcome after acute ischaemic stroke: an analysis of data from the Interventional Management of Stroke (IMS III) phase 3 trial. Lancet Neurol 2014;13:567-74.

11 Lansberg MG, Dabus G. Interaction between time to treatment and reperfusion therapy in patients with acute ischemic stroke. J Neurointerv Surg 2013;5(Suppl 1):i48-51.

12 Mazighi $\mathrm{M}$, Meseguer $\mathrm{E}$, Labreuche J, et al. Dramatic recovery in acute ischemic stroke is associated with arterial recanalization grade and speed. Stroke 2012:43:2998-3002.

13 The Penumbra Pivotal Stroke Trial Investigators. The Penumbra Pivotal Stroke Trial. Safety and effectiveness of a new generation of mechanical devices for clot removal in intracranial large vessel occlusive disease. Stroke 2009:40:2761-8.

14 Frei D, Gerber J, Turk A, et al. The SPEED study: initial clinical evaluation of the Penumbra novel 054 reperfusion catheter. J Neurointerv Surg 2013;5(Suppl 1): i74-6.

15 Almekhlafi MA, Eesa M, Menon BK, et al. Ultrashort imaging to reperfusion time interval arrests core expansion in endovascular therapy for acute ischemic stroke. J Neurointerv Surg 2013:5(Suppl 1):i58-61.

16 Sheth SA, Jahan R, Gralla J, et al. Time to endovascular reperfusion and degree of disability in acute stroke. Ann Neurol 2015;78:584-93.

17 Broderick JP, Palesch YY, Demchuk AM, et al. Endovascular therapy after intravenous t-PA versus t-PA alone for stroke. N Engl J Med 2013;368: 893-903.

18 Mocco J, Fiorella D, Fargen KM, et al. Endovascular therapy for acute ischemic stroke is indicated and evidence based: a position statement. J Neurointerv Surg 2015;7:79-81.

19 Fransen PS, Berkhemer OA, Lingsma HF, et al. Multicenter randomized clinical trial of endovascular treatment of acute ischemic stroke in the Netherlands Investigators. Time to reperfusion and treatment effect for acute ischemic stroke: a randomized clinical trial. JAMA Neurol 2016;73:190-6.

20 Menon BK, Sajobi TT, Zhang Y, et al. Analysis of workflow and time to treatment on thrombectomy outcome in the ESCAPE randomized controlled trial. Circulation 2016;133:2279-86.

21 Goyal M, Jadhav AP, Bonafe A, et al., SWIFT PRIME investigators. Analysis of workflow and time to treatment and the effects on outcome in endovascular treatment of acute ischemic stroke: results from the SWIFT PRIME randomized controlled trial. Radiology 2016;279:888-97.

22 Gupta R, Xiang B, Ge S, et al. Stagnation of treatment times over a decade: results of a pooled analysis from the MERCI registry, MERCI, TREVO, and TREVO 2 trials. J Neurointerv Surg 2016;8:453-6.

23 Liebeskind DS, Jahan R, Nogueira RG, et al. Early arrival at the emergency department is associated with better collaterals, smaller established infarcts and better clinical outcomes with endovascular stroke therapy: SWIFT study. J Neurointerv Surg 2016;8:553-8.

24 Powers WJ, Derdeyn CP, Biller J, et al.; American Heart Association Stroke Council. 2015 American Heart Association/American Stroke Association Focused Update of the 2013 Guidelines for the Early Management of Patients With Acute Ischemic Stroke Regarding Endovascular Treatment: A Guideline for Healthcare Professionals From the American Heart Association/American Stroke Association. Stroke 2015;46:3020-35

25 Goyal M, Almekhlafi MA, Fan L, et al. Evaluation of interval times from onset to reperfusion in patients undergoing endovascular therapy in the Interventional Management of Stroke III trial. Circulation 2014;130:265-72.

26 Gorelick PB. Primary and comprehensive stroke centers: history, value and certification criteria. J Stroke 2013;15:78-89.

27 Fedder W. National and international quality initiatives to improve stroke care. Neurol Clin 2008;26:1191-207, xi.

28 Lichtman JH, Jones SB, Wang Y, et al. Outcomes after ischemic stroke for hospitals with and without Joint Commission-certified primary stroke centers. Neurology 2011;76:1976-82.

29 Lichtman JH, Jones SB, Leifheit-Limson EC, et al. 30-Day mortality and readmission after hemorrhagic stroke among Medicare beneficiaries in Joint Commission primary stroke center-certified and noncertified hospitals. Stroke 2011:42:3387-91.

30 Rajamani K, Millis S, Watson S, et al. Thrombolysis for acute ischemic stroke in Joint Commission-certified and -noncertified hospitals in Michigan. J Stroke Cerebrovasc Dis 2013;22:49-54.

31 Alberts MJ, Latchaw RE, Selman WR, et al. Recommendations for comprehensive stroke centers: a consensus statement from the Brain Attack Coalition. Stroke 2005;36:1597-616.

32 Stroke Certification Programs-Program Concept Comparison. jointcommission.org (published 21 July 2015). http://www.jointcommission.org/assets/1/18/ StrokeProgramGrid_abbr_AHA-TJC_5-1-15.pdf (accessed 21 Jul 2015).

33 McKinney JS, Cheng JQ, Rybinnik I, et al. Myocardial Infarction Data Acquisition System (MIDAS 22) Study Group. Comprehensive stroke centers may be associated with improved survival in hemorrhagic stroke. J Am Heart Assoc 2015;4: e001448.

34 Cramer SC, Stradling D, Brown DM, et al. Organization of a United States county system for comprehensive acute stroke care. Stroke 2012;43: 1089-93.

35 Higashida R, Alberts MJ, Alexander DN, et al. Interactions within stroke systems of care: a policy statement from the American Heart Association/American Stroke Association. Stroke 2013:44:2961-84.

36 Leifer D, Bravata DM, Connors JJB, et al. Metrics for measuring quality of care in comprehensive stroke centers: detailed follow-up to Brain Attack Coalition comprehensive stroke center recommendations: a statement for healthcare professionals from the American Heart Association/American Stroke Association. Stroke 2011;42:849-77. 
37 Schuberg S, Song S, Saver JL, et al. Impact of emergency medical services stroke routing protocols on Primary Stroke Center certification in California. Stroke 2013;44:3584-6.

38 Tissue plasminogen activator for acute ischemic stroke. The National Institute of Neurological Disorders and Stroke rt-PA Stroke Study Group. N Engl J Med 1995:333:1581-7.

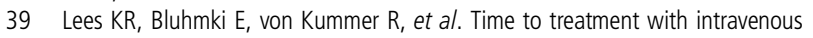
alteplase and outcome in stroke: an updated pooled analysis of ECASS, ATLANTIS, NINDS, and EPITHET trials. Lancet 2010;375:1695-703.

40 Harbison J, Hossain 0 , Jenkinson D, et al. Diagnostic accuracy of stroke referrals from primary care, emergency room physicians, and ambulance staff using the face arm speech test. Stroke 2003;34:71-6.

41 Kothari RU, Pancioli A, Liu T, et al. Cincinnati Prehospital Stroke Scale: reproducibility and validity. Ann Emerg Med 1999;33:373-8.

42 Kidwell CS, Saver JL, Schubert GB, et al. Design and retrospective analysis of the Los Angeles Prehospital Stroke Screen (LAPSS). Prehosp Emerg Care 1998:2:267-73.

43 Kidwell CS, Starkman S, Eckstein M, et al. Identifying stroke in the field: prospective validation of the Los Angeles prehospital stroke screen (LAPSS). Stroke 2000;31:71-6.

44 Kleindorfer DO, Miller R, Moomaw CJ, et al. Designing a message for public education regarding stroke: does FAST capture enough stroke? Stroke 2007;38:2864-8.

45 Wall HK, Beagan BM, O'Neill J, et al. Addressing stroke signs and symptoms through public education: the Stroke Heroes Act FAST campaign. Prev Chronic Dis 2008;5:A49.

46 Pierce C, Fahs PS, Dura A, et al. Raising stroke awareness among rural dwellers with a Facts for Action to Stroke Treatment-based educational program. App/ Nurs Res 2011;24:82-7.

47 Kleindorfer D, Miller R, Sailor-Smith S, et al. The challenges of community-based research: the beauty shop stroke education project. Stroke 2008;39:2331-5.

48 Wolters FJ, Paul NLM, Li L, et al. Oxford Vascular Study. Sustained impact of UK FAST-test public education on response to stroke: a population-based time-series study. Int J Stroke 2015;10:1108-14.

49 Minnerup J, Wersching $\mathrm{H}$, Unrath $\mathrm{M}$, et al. Effects of emergency medical service transport on acute stroke care. Eur J Neurol 2014;21:1344-7.

50 Adeoye 0, Albright KC, Carr BG, et al. Geographic access to acute stroke care in the United States. Stroke 2014;45:3019-24.

51 Curfman D, Connor LT, Moy HP, et al. Accuracy of emergency medical services-reported last known normal times in patients suspected with acute stroke. Stroke 2014:45:1275-9.

52 Bray JE, Martin J, Cooper G, et al. Paramedic identification of stroke: community validation of the Melbourne Ambulance Stroke Screen. Cerebrovasc Dis 2005;20:28-33.

53 Studnek JR, Asimos A, Dodds J, et al. Assessing the validity of the Cincinnati prehospital stroke scale and the medic prehospital assessment for code stroke in an urban emergency medical services agency. Prehosp Emerg Care 2013:17:348-53.

54 Nor AM, Davis J, Sen B, et al. The Recognition of Stroke in the Emergency Room (ROSIER) scale: development and validation of a stroke recognition instrument. Lancet Neurol 2005;4:727-34.

55 Jauch EC, Saver JL, Adams HP, et al. Guidelines for the early management of patients with acute ischemic stroke: a guideline for healthcare professionals from the American Heart Association/American Stroke Association. Stroke 2013;44:870-947.

56 Asimos AW, Ward S, Brice JH, et al. Out-of-hospital stroke screen accuracy in a state with an emergency medical services protocol for routing patients to acute stroke centers. Ann Emerg Med 2014;64:509-15.

57 Oostema JA, Konen J, Chassee T, et al. Clinical predictors of accurate prehospital stroke recognition. Stroke 2015;46:1513-17.

58 Brandler ES, Sharma M, McCullough F, et al. Prehospital stroke identification: factors associated with diagnostic accuracy. I Stroke Cerebrovasc Dis 2015;24:2161-6.

59 Grotta JC, Savitz SI, Persse D. Stroke severity as well as time should determine stroke patient triage. Stroke 2013;44:555-7.

60 Singer OC, Dvorak F, du Mesnil de Rochemont R, et al. A simple 3-item stroke scale: comparison with the National Institutes of Health Stroke Scale and prediction of middle cerebral artery occlusion. Stroke 2005;36:773-6.

61 Nazliel B, Starkman S, Liebeskind DS, et al. A brief prehospital stroke severity scale identifies ischemic stroke patients harboring persisting large arterial occlusions. Stroke 2008:39:2264-7.

62 Katz BS, McMullan JT, Sucharew $\mathrm{H}$, et al. Design and validation of a prehospital scale to predict stroke severity: Cincinnati Prehospital Stroke Severity Scale. Stroke 2015:46:1508-12.

63 Pérez de la Ossa N, Carrera D, Gorchs M, et al. Design and validation of a prehospital stroke scale to predict large arterial occlusion: the rapid arterial occlusion evaluation scale. Stroke 2014;45:87-91.

64 Fischer U, Arnold M, Nedeltchev K, et al. NIHSS score and arteriographic findings in acute ischemic stroke. Stroke 2005;36:2121-5.
65 Heldner MR, Zubler C, Mattle HP, et al. National Institutes of Health stroke scale score and vessel occlusion in 2152 patients with acute ischemic stroke. Stroke 2013;44:1153-7.

66 Gupta R, Manuel M, Owada K, et al. Severe hemiparesis as a prehospital tool to triage stroke severity: a pilot study to assess diagnostic accuracy and treatment times. J Neurointerv Surg 2016;8:775-7.

67 Venizelos A, Chen S, Gianatasio R, et al. Abstract T MP59: Texas stroke intervention pre-hospital stroke severity scale (aka LEGS score): a triaging tool for interventional stroke therapy. Stroke 2014;45(Suppl 1):ATMP59.

68 Teleb MS, Ver Hage A, Carter J, et al. Stroke vision, aphasia, neglect (VAN) assessment-a novel emergent large vessel occlusion screening tool: pilot study and comparison with current clinical severity indices. J Neurointerv Surg Published Online First: 17 Feb 2016. doi:10.1136/neurintsurg-2015-012131

69 Tirschwell DL, Longstreth WT, Becker KJ, et al. Shortening the NIH Stroke scale for use in the prehospital setting. Stroke 2002;33:2801-6.

70 Gonzalez MA, Hanna N, Rodrigo ME, et al. Reliability of prehospital real-time cellular video phone in assessing the simplified National Institutes of Health Stroke Scale in patients with acute stroke: a novel telemedicine technology. Stroke 2011:42:1522-7.

71 Hölscher T, Schlachetzki F, Zimmermann M, et al. Transcranial ultrasound from diagnosis to early stroke treatment. 1. Feasibility of prehospital cerebrovascular assessment. Cerebrovasc Dis 2008;26:659-63.

72 Schlachetzki F, Herzberg M, Hölscher T, et al. Transcranial ultrasound from diagnosis to early stroke treatment: part 2: prehospital neurosonography in patients with acute stroke: the Regensburg stroke mobile project. Cerebrovasc Dis 2012:33:262-71.

73 Hölscher T, Dunford JV, Schlachetzki F, et al. Prehospital stroke diagnosis and treatment in ambulances and helicopters-a concept paper. Am J Emerg Med 2013:31:743-7.

74 Herzberg M, Boy S, Hölscher T, et al. Prehospital stroke diagnostics based on neurological examination and transcranial ultrasound. Crit Ultrasound J 2014;6:3.

75 Fassbender K, Walter $\mathrm{S}$, Liu Y, et al. "Mobile stroke unit" for hyperacute stroke treatment. Stroke 2003;34:e44.

76 Walter S, Kostpopoulos P, Haass A, et al. Bringing the hospital to the patient: first treatment of stroke patients at the emergency site. PLOS ONE 2010;5:e13758.

77 Rajan SS, Baraniuk S, Parker S, et al. Implementing a mobile stroke unit program in the United States: why, how, and how much? JAMA Neurol 2015;72: 229-34.

78 Parker SA, Bowry R, Wu TC, et al. Establishing the first mobile stroke unit in the United States. Stroke 2015;46:1384-91.

79 Klein KE, Rasmussen PA, Winners SL, et al. Teleneurocritical care and telestroke. Crit Care Clin 2015;31:197-224.

80 Ebinger $M$, Kunz $A$, Wendt $M$, et al. Effects of golden hour thrombolysis: a Prehospital Acute Neurological Treatment and Optimization of Medical Care in Stroke (PHANTOM-S) substudy. JAMA Neurol 2015;72:25-30.

81 Cerejo $R$, John $S$, Buletko $A B$, et al. A mobile stroke treatment unit for field triage of patients for intraarterial revascularization therapy. J Neuroimaging 2015;25: 940-5.

82 Dietrich M, Walter S, Ragoschke-Schumm A, et al. Is prehospital treatment of acute stroke too expensive? An economic evaluation based on the first trial. Cerebrovasc Dis 2014;38:457-63.

83 Prabhakaran S, O'Neill K, Stein-Spencer L, et al. Prehospital triage to primary stroke centers and rate of stroke thrombolysis. JAMA Neurol 2013;70: 1126-32.

84 McKinney JS, Mylavarapu K, Lane J, et al. Hospital prenotification of stroke patients by emergency medical services improves stroke time targets. J Stroke Cerebrovasc Dis 2013;22:113-18.

85 Patel MD, Rose KM, O'Brien EC, et al. Prehospital notification by emergency medical services reduces delays in stroke evaluation: findings from the North Carolina stroke care collaborative. Stroke 2011;42:2263-8.

86 Binning MJ, Sanfillippo G, Rosen W, et al. The neurological emergency room and prehospital stroke alert: the whole is greater than the sum of its parts. Neurosurgery 2014;74:281-5; discussion 285

87 Oostema JA, Nasiri M, Chassee T, et al. The quality of prehospital ischemic stroke care: compliance with guidelines and impact on in-hospital stroke response. J Stroke Cerebrovasc Dis 2014;23:2773-9.

88 de la Ossa NP, Sánchez-Ojanguren J, Palomeras E, et al. Influence of the stroke code activation source on the outcome of acute ischemic stroke patients. Neurology 2008;70:1238-43.

89 Fuentes B, Alonso de Leciñana M, Ximénez-Carrillo A, et al. Futile interhospital transfer for endovascular treatment in acute ischemic stroke: the Madrid stroke network experience. Stroke 2015;46:2156-61.

90 Crocco TJ, Grotta JC, Jauch EC, et al. EMS management of acute strokeprehospital triage (resource document to NAEMSP position statement). Prehosp Emerg Care 2007;11:313-17.

91 Prabhakaran S, Ward E, John S, et al. Transfer delay is a major factor limiting the use of intra-arterial treatment in acute ischemic stroke. Stroke 2011;42:1626-30. 
92 Froehler M, Espaillat K. P-001 evaluation of transfer times for emergent stroke patients from regional centers to a comprehensive center. J Neurointerv Surg 2015;7(Suppl 1):A21-2.

93 Southerland AM, Johnston KC, Molina CA, et al. Suspected large vessel occlusion: should emergency medical services transport to the nearest primary stroke center or bypass to a comprehensive stroke center with endovascular capabilities? Stroke 2016;47:1965-7.

94 LaBuzetta JN, Yoo AJ, Ali S, et al. Determinants of early outcomes in patients with acute ischemic stroke and proximal artery occlusion. J Stroke Cerebrovasc Dis 2014;23:2527-32

95 Doumouras AG, Haas B, Gomez D, et al. The impact of distance on triage to trauma center care in an urban trauma system. Prehosp Emerg Care 2012;16:456-62.

96 Terkelsen $C J$, Pinto D, Clemmensen $P$, et al. A response to a misrepresentation of the STEMI guidelines: the response. Heart 2013;99:1787-8.

97 Jayaraman MV, Iqbal A, Silver B, et al. Developing a statewide protocol to ensure patients with suspected emergent large vessel occlusion are directly triaged in the field to a comprehensive stroke center: how we did it. J Neurointerv Surg Published Online First: 3 Mar 2016. doi:10.1136/neurintsurg-2016-012275

98 Fosbol EL, Granger CB, Jollis JG, et al. The impact of a statewide pre-hospital STEMI strategy to bypass hospitals without percutaneous coronary intervention capability on treatment times. Circulation 2013;127:604-12.

99 Widimsky P, Bilkova D, Penicka M, et al. Long-term outcomes of patients with acute myocardial infarction presenting to hospitals without catheterization laboratory and randomized to immediate thrombolysis or interhospital transport for primary percutaneous coronary intervention. Five years' follow-up of the PRAGUE-2 Trial. Eur Heart J 2007;28:679-84.

100 Sun $\mathrm{CH}$, Nogueira RG, Glenn BA, et al. "Picture to puncture": a novel time metric to enhance outcomes in patients transferred for endovascular reperfusion in acute ischemic stroke. Circulation 2013;127:1139-48.

101 Sheth KN, Smith EE, Grau-Sepulveda MV, et al. Drip and ship thrombolytic therapy for acute ischemic stroke: use, temporal trends, and outcomes. Stroke 2015:46:732-9.
102 Asaithambi G, Chaudhry SA, Hassan AE, et al. Adherence to guidelines by emergency medical services during transport of stroke patients receiving intravenous thrombolytic infusion. J Stroke Cerebrovasc Dis 2013; 22:e42-5.

103 Krumholz HM, Anderson JL, Bachelder BL, et al. ACC/AHA 2008 performance measures for adults with ST-elevation and non-ST-elevation myocardial infarction: a report of the American College of Cardiology/American Heart Association Task Force on Performance Measures (Writing Committee to develop performance measures for ST-elevation and non-ST-elevation myocardial infarction): developed in collaboration with the American Academy of Family Physicians and the American College of Emergency Physicians: endorsed by the American Association of Cardiovascular and Pulmonary Rehabilitation, Society for Cardiovascular Angiography and Interventions, and Society of Hospital Medicine. Circulation 2008;118:2596-648

104 van Diepen S, Widimsky P, Lopes RD, et al. Transfer times and outcomes in patients with ST-segment-elevation myocardial infarction undergoing interhospital transfer for primary percutaneous coronary intervention: APEX-AMI insights. Circ Cardiovasc Qual Outcomes 2012;5:437-44.

105 Svenson JE, O'Connor JE, Lindsay MB. Is air transport faster? A comparison of air versus ground transport times for interfacility transfers in a regional referral system. Air Med J 2006;25:170-2.

106 McVey J, Petrie DA, Tallon JM. Air versus ground transport of the major trauma patient: a natural experiment. Prehosp Emerg Care 2010;14:45-50.

107 Baylous D, Tillman HJ, Smith MW. Air versus ground transport for patients with ST-elevation myocardial infarction: does transport type affect patient outcomes? J Emerg Nurs 2013;39:e65-74.

108 Silliman SL, Quinn B, Huggett V, et al. Use of a field-to-stroke center helicopter transport program to extend thrombolytic therapy to rural residents. Stroke 2003;34:729-33.

109 Lukovits TG, Von Iderstine SL, Brozen R, et al. Interhospital helicopter transport for stroke. Air Med J 2013;32:36-9.

110 Rubin MN, Demaerschalk BM. The use of telemedicine in the management of acute stroke. Neurosurg Focus 2014;36:E4. 